\title{
Is Schrodinger Equation a Relativistic Effect?
}

\section{Koutandos S*}

Ekfe Chiou, Agia Paraskevi, Greece

*Corresponding author: Spiros Koutandos, EKFE CHIOU, Maniakiou 17, Agia Paraskevi, Attica 15343, Greece, Tel: 6980129686; Email: skoutandos1@gmail.com

\section{Research Article}

Volume 5 Issue 1

Received Date: March 03, 2021

Published Date: March 18, 2021

DOI: $10.23880 / p s b j-16000165$

\section{Abstract}

We prove that associated with mass is spacetime curvature which leads to creation of volume and mass shells in every instance due to the energy equivalence principle. We put forth the relations between a point surface tension and a spacetime curvature. The conclusion is that the spacetime is of fluid nature. Finally we find the chi psi omega law.

Keywords: General relativity; Special relativity; Quantum thermodynamics; Quantum mechanics

\section{Introduction}

In a series of published papers of Koutandos S [1-3] we have proved London equations and Ginzburg-Landau expansion of free energy in superconductors based on the assumption that the presence of mass induces a constant curvature $\mathrm{K}$ in spacetime and this together with the charge alters the speed of light by introducing a dielectric susceptibility chi. In the case of one electron $\mathrm{K}$ is associated with the Compton wavelength and the fine structure constant by considerations of the relativistic radius of the electron:

$$
\begin{gathered}
\frac{m c^{2}}{R}=\frac{e^{2}}{4 \pi \varepsilon_{0} R^{2}}=K m c^{2} \\
\alpha=\frac{e^{2}}{4 \pi \varepsilon_{0} \hbar c}(2) \\
\lambda_{c}=\frac{\hbar}{m c}
\end{gathered}
$$

From equations (1), (2) and (3) we may evaluate $\mathrm{K}$

$$
K=\frac{1}{\alpha \lambda_{c}}(4)
$$

\section{Main Part}

We may borrow a term called mass shell constraint from general relativity [4] which comes from the following rule:

$$
g^{\mu v} p_{\mu} p_{v}=m c^{2}(5)
$$

In formula (5) $\mathrm{p}$ stands for momentum. The time equivalent of momentum in relativity is energy. In the formula we shall put forth it is the energy minus the potential energy times the probability:

$$
\frac{\hbar^{2}}{2 m N} \Delta|\psi|^{2}=\frac{|\psi|^{2}}{N}(E-U)-\frac{\vec{p}^{*} \cdot \vec{p}}{m N}=\frac{m c^{2}}{N \chi}=\frac{m \bar{c}^{2}}{N}
$$

Equation (6) is in accordance with the Takabayashi tensor [5] of internal stress. Equation (6) implies a variable speed of light as was Einstein's first idea [6] in the beginning of his writings regarding general relativity. He suggested back then that a distribution of mass would alter the speed of light.

We have given a literal definition of the mass shell since we hypothesize that mass resides on surfaces and creates a surface tension with the energy taken from the equivalence principle creating new surface and volume:

$$
\frac{\hbar^{2}}{2 m} \Delta P=B=\gamma K=\frac{m c^{2}}{N \chi}=V \frac{d P}{d V}(7)
$$

In formula (7) gamma stands for a point surface tension and $\mathrm{B}$ is the bulk modulus. This formula is a reformulation of the classical one connecting the speed of sound which is longitudinal wave with the bulk modulus. In the case of 
superfluids it is successful. From the mass energy equivalence principle we presume that the surface density of energy or else surface tension is associated with a mass surface density as has been mentioned by other authors as well [7]:

$$
\gamma=c^{2} \frac{d m}{d S}(8)
$$

From the aforementioned we conclude that:

$$
K \chi(\vec{r})=\frac{d S}{d V}(9)
$$

That is to say the total curvature defines the surface to volume ratio.

The spacetime metric should change having thus a space dependent light velocity. This idea has already been implemented in previous work [4]. We produce the formula:

$$
d s^{2}=\frac{c^{2}}{\chi(\vec{r})} d t^{2}-d \vec{r}^{2}(10)
$$

We believe that the total time derivative of the radius $[1,4]$ vector is different from the velocity because the particle exists in a fluid spacetime therefore the different quantities transform according to the following relationships:

$$
\begin{gathered}
\frac{d \vec{r}}{d t}=\frac{\hbar}{m} \nabla \varphi+\frac{e}{m c} \vec{A} \\
\frac{d f}{d t}=\nabla f \cdot \frac{d \vec{r}}{d t} \\
\frac{d \vec{A}}{d t}=\left(\left(\frac{d \vec{r}}{d t}\right) \cdot \nabla\right) \vec{A}
\end{gathered}
$$

The London equations for superconductors are a natural consequence of equations (11), (12), (13) together with the fact that the potential energy is purely of magnetic nature in that case. As an intermediate we find that there is a point flux phi equal to the phase of the wave function. These results describe turbulent surfaces with a pressure jump and a Coriolis force is added which is as follows:

$$
\begin{gathered}
\vec{\Omega}=\nabla \times \bar{J} \\
\vec{V}_{\text {coriolis }}=\vec{\Omega} \times \frac{d \vec{r}}{d t}(15)
\end{gathered}
$$

The aforementioned results can be found in a theses [8] describing vortices in a superfluid if we interpret these outcomes as a general rule for the quantum particle as a spherical vortex entity. There is also a rich literature on the subject of fluid spacetime [9-13]. According to the findings of this theory the transformations of equations (11),(12),(13) are the result of a mass field which changes the volume.

In previous work [1] the Bohm potential or quantum potential as is known was calculated:

$$
\frac{\hbar^{2}}{2 m} \frac{\Delta|\psi|}{|\psi|}=E-U-\frac{m}{2}\left(\frac{d \vec{r}}{d t}\right)^{2}=Q
$$

Now we assume that:

$$
\begin{array}{r}
-\frac{\hbar^{2}}{2 m}|\psi| \Delta|\psi|=-\chi \Omega \\
\Omega=-P V(18)
\end{array}
$$

Gathering formulas (7),(15),(16),(17) we arrive at the following equation:

$$
\frac{P d V}{V d P}=\frac{Q}{m c^{2}} \frac{N}{V}=\left(E-U-\frac{1}{2} m\left(\frac{d \vec{r}}{d t}\right)^{2}\right) \frac{N}{m c^{2} V}
$$

We also know [3] that:

$$
P=\frac{|\psi|^{2}}{N}(E-U)
$$

In equation (19) volume can be omitted from both sides and we do not have undesirable effects in integration.

\section{Conclusion}

Formula (17) should be called the chi psi omega law after the last three letters of the Greek alphabet. In the case where the wave function does not have a phase or there is no magnetic field $\mathrm{Q}=\mathrm{E}-\mathrm{U}$ and we can deduce from equations (19), (20)

$$
\delta P=\frac{\delta(m) c^{2}}{N}
$$

Therefore mass fluctuates the volume and induces an overall pressure on the system.

\section{References}

1. Koutandos S (2020) About a possible derivation of the London equations. Physics Essays 33(2): 208-210.

2. Koutandos S (2020) A discussion about the hidden variables of quantum mechanics. Physics Essays 33(4): 513-514. 
3. Koutandos S (2021) Expalining Ginzburg Landau equations from first principles. Phys Sci \& Biophys J 5(1): 1-3.

4. Alsing PM, Evans JC, Nandi KM (2000) The phase of a quantum mechanical particle in curved spacetime. General Relativity and Gravitation 33: 1459-1487.

5. Takabayashi $\mathrm{T}$ (1953) Remarks on the formulation of quantum mechanics with classical pictures and on relations between linear scalar fields and hydrodynamical fields. Progress of Theoretical Physics 9(3): 187-222.

6. Einstein A (1907) On the relativity principle and the conclusions drawn from it. Jahrbuch der Elektrizit"at und Elektronik 4: 411-462.

7. Bressange GF (2000) On the extension of the concept of thin shells to the Einstein-Cartan theory. Classical and Quantum Gravity 17(13): 2509-2523.
8. Thompson L (2010) Equation of motion of a quantum vortex. Theses for the University of Columbia (Vancouver), USA.

9. Beig R, Simon W (1992) On the uniqueness of static perfect-fluid solutions in general relativity. Communications in Mathematical physics 144: 373-390.

10. Obunkhov YN (2001) Spin gravty and inertia. Phys Rev Lett 86(2): 192-195.

11. Mallick S, Jin Suh Y, Chand De U (2016) A spacetime with pseudo-projective curvature tensor. Journal of Mathematical Physics 57(6): 062501.

12. Millette PA (2014) Wave-Particle Duality in the Elastodynamics of the Spacetime Continuum (STCED). Progress in Physics 10(4): 255-258.

13. Smerlak M (2012) Diffusion in curved spacetimes. New J Phys 14: 023019. 